Monatsschr Kinderheilkd

2020 · 168 (Suppl 3):S197-S203

https://doi.org/10.1007/s00112-020-01014-7

Online publiziert: 20. August 2020

๑) Der/die Autor(en) 2020

\section{Die Innsbrucker Kinderklinik vor Asperger}

Am 28. Juni 1934 wurde Johann Loos (1863-1943), seit 1896 erster Extraordinarius bzw. seit 1911 erster Ordinarius der Innsbrucker Kinderklinik, mit Ende September 1934 aus Altersgründen in den "dauernden Ruhestand“ versetzt. ${ }^{1}$ Seine größte Errungenschaft bestand in der Eröffnung des eigenständigen Kinderklinikpavillons am 24. Juni 1901; vorher mussten die pädiatrischen PatientInnen in Räumlichkeiten der inneren $\mathrm{Me}$ dizin betreut werden [7, S. 82].

Loos' Nachfolger wurde der in Wien geborene Richard Priesel (1890-1955; - Abb. 1). Dieser absolvierte ebendort sein Medizinstudium und wurde bereits im Jänner 1915, nur einen Monat nach seiner Promotion, zum Kriegsdienst eingezogen. Letztlich sollte Priesel als Truppenarzt bis Kriegsende eingesetzt bleiben. Nach dem Ersten Weltkrieg begann er seine klinische Ausbildung am Krankenhaus Wieden in Wien, bevor er 1923 als Hilfsarzt an die Universitätskinderklinik Wien wechselte. Bereits ab 1925 war er dort unter Clemens von Pirquet (1874-1929) als Assistent angestellt und blieb dies für zehn Jahre. Während seiner Zeit dort lernte Priesel auch Hans Asperger kennen (• Abb. 2). Eine wissenschaftliche Zusammenarbeit

\footnotetext{
1 Universitätsarchiv Innsbruck(UAI), Lehrkanzel für Kinderheilkunde nach Prof. Loos 1934/1935, Schreiben der Landeshauptmannschaft für Tirol an Johann Loos vom 10.08.1934.
}

Dieser Beitrag wurde u. a. durch zur Verfügung gestellte Literatur aus der Sammlung des medizinhistorischen Vereins "Freundeskreis Pesthaus" ermöglicht.

\title{
C. Lechner
}

Department für Kinder- und Jugendheilkunde, Pädiatrie I, Medizinische Universität Innsbruck, Innsbruck, Österreich

\section{Hans Asperger und die Kinderklinik Innsbruck}

der beiden ist zumindest aus den jeweiligen Publikationsverzeichnissen nicht ersichtlich. Dafür dürften die fachlichen Schwerpunkte, Priesels auf dem Gebiet des Diabetes mellitus bei Kindern und Jugendlichen, Aspergers bekanntermaßen auf der Heilpädagogik, zu unterschiedlich gewesen sein.

Priesel hat allerdings fast die Hälfte seiner Publikationen gemeinsam mit dem Wiener Pädiater und Diabetesspezialisten Richard Wagner (1887-1974) veröffentlicht. Dieser setzte als Erster in Österreich Insulin zur Behandlung des Diabetes mellitus bei Kindern ein und fasste seine ersten Ergebnisse bereits 1925 für die Zeitschrift für Kinderheilkunde zusammen [10]. Das NS-Regime kategorisierte Wagner als Juden und erkannte ihm die Lehrbefugnis ab. Noch 1938 konnte er in die USA nach Boston emigrieren und dort seine Forschung fortsetzen [8, S. 404].

\section{》) Ein gutes Jahr nach der Habilitation wurde Priesel zum Vorstand der Innsbrucker Kinderklinik ernannt}

Im Juni 1934 konnte sich Priesel schließlich unter dem Klinikdirektor und illegalen Nationalsozialisten Franz Hamburger (1874-1954) habilitieren. Nur ein gutes Jahr später erfolgte am 27. September 1935 die Ernennung zum „außerordentlichen Professor der Kinderheilkunde" und damit zum Vorstand der Inns- brucker Kinderklinik. ${ }^{2}$ Seinen Dienst in Innsbruck begann Priesel am 1. Oktober 1935.

Nach dem sog. Anschluss trat Priesel dem Nationalsozialistischen Deutschen Ärztebund (NSD-Ärztebund) bei und stellte einen Aufnahmeantrag für den NSD-Dozentenbund. ${ }^{3}$ Anwärter oder gar Mitglied der Nationalsozialistischen Deutschen Arbeiterpartei (NSDAP) wurde er jedoch nicht. ${ }^{4}$ Laut Bericht des "Sicherheitsdienst[es] des RF [Reichsführer] SS“ wurde Priesel als „politisch nicht interessiert" eingestuft [4, S. 58].

Wenige Wochen später erfolgte dennoch vonseiten des Dekans (und NSDAP-Mitglieds) Franz Josef Lang (1894-1975) der Antrag an das Ministerium um Ernennung Priesels zum Ordinarius. ${ }^{5}$ Diesen Antrag unterstützte auch der damalige Rektor und langjährige NS-Sympathisant Harold Steinacker (1875-1965). Offenbar aufgrund ausbleibender Rückmeldungen beantragte das Dekanat im Oktober 1939 und im Februar 1940 erneut die Beförderung zum ordentlichen Professor. Mit Schreiben vom 23. April 1940 gab die verantwortliche

2 UAl, Lehrkanzel für Kinderheilkunde nach Prof. Loos, Schreiben des Staatssekretärs Loebenstein an das Dekanat der medizinischen Fakultät der Universität vom 05.10.1935.

3 UAl, Personalakt Richard Priesel, Vorschlag zur Ernennung an den Herrn Staatsminister und Chef der Präsidialkanzlei des Führers und Reichskanzlers vom 15.04.1941.

4 UAl, Personalakt Richard Priesel, Personalnachrichten vom 10.04.1942.

5 UAl, Personalakt Richard Priesel, Dekan der medizinischen Fakultät an das Ministerium für innere und kulturelle Angelegenheiten (Abt. IV, Erziehung, Kultus und Volksbildung) vom 31.10.1939. 


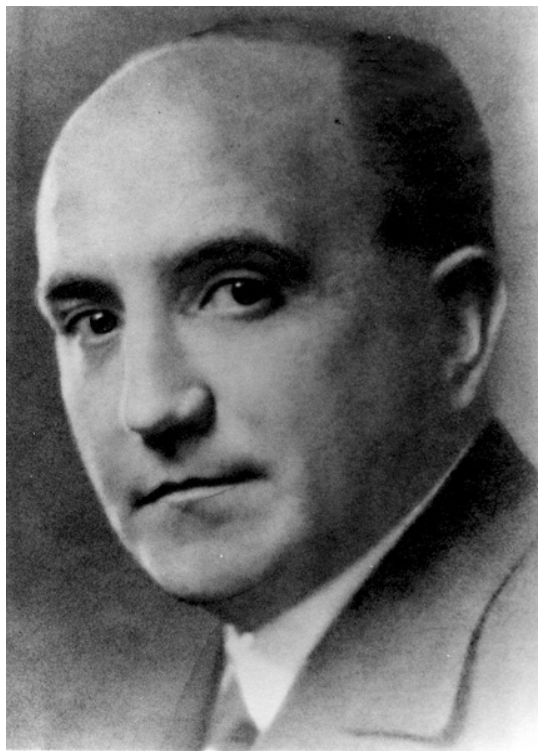

Abb. $1 \Delta$ Porträtfoto von Richard Priesel. (@ Archiv Kinderklinik Innsbruck)

Abteilung in Wien kund, dass grundsätzlich „nach dem Dienstpostenplan der Innsbrucker Universitätsprofessoren [...] eine ordentliche Lehrkanzel zur Verfügung steht". ${ }^{6}$ Wenige Tage vorher erhielt der Kurator der Universität die gegenteilige Mitteilung aus dem Reichsministerium für Wissenschaft, Erziehung und Volksbildung, dass es während des Krieges „ausgeschlossen erscheint, die Umwandlung des Extraordinariats in ein Ordinariat beim Reichsfinanzminister zu erreichen", auch dass eine freie Planstelle nicht zur Verfügung stehen würde. ${ }^{7}$ Mit 1. August 1942 wurde Priesel dennoch zum Ordinarius ernannt. ${ }^{8}$ Bereits 1939 erfolgte vonseiten des Dekanats der Antrag auf Erhöhung des Diensteinkommens, welches unter dem

${ }^{6}$ UAI, Personalakt Richard Priesel, Schreiben des Ministeriums für innere und kulturelle Angelegenheiten (Abt. IV, Erziehung, Kultus u. Volksbildung) an den Kurator der Universität Innsbruck vom 23.04.1940.

7 UAl, Personalakt Richard Priesel, Schreiben des Reichsministers für Wissenschaft, Erziehung und Volksbildung an den Kurator der Universität Innsbruck vom 15.04.1940.

8 UAl, Personalakt Richard Priesel, Lebenslauf, ohne Datum.

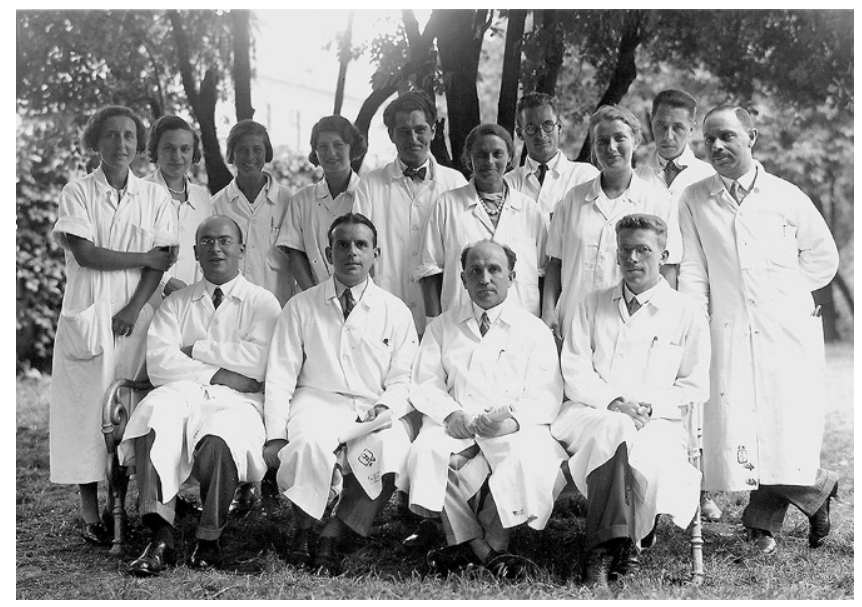

Abb. $2<$ Personal der Wiener Universitäts-Kinderklinik, Hans Asperger vordere Reihe ganz rechts im Bild, links neben ihm Richard Priesel, 1933. (@ Josephinum-Ethik, Sammlungen und Geschichte der Medizin, MedUni Wien)

des „1. Assistent[en] der Klinik, Dozent Dr. Niederwieser" lag. ${ }^{\text {" }}$

Priesels persönliche Einstellung zum NS-Regime bleibt aus den vorhandenen Quellen schwer nachvollziehbar. Es gibt derzeit keine Hinweise auf medizinische Versuche an Kindern und Jugendlichen an der Innsbrucker Kinderklinik während der Zeit des Nationalsozialismus, wie sie etwa an der Wiener Kinderklinik geschahen. Eine systematische Aufarbeitung für den Standort Innsbruck steht allerdings noch aus.

Während des Zweiten Weltkrieges litt auch die Kinderklinik unter anhaltenden Versorgungsengpässen. Verschärft wurde die Situation durch die im Rahmen der Schutzmaßnahmen teilweise ausgelagerten PatientInnen in Fulpmes und dem Volderwaldhof bei Hall in Tirol. Zudem traf am 25. Dezember 1943 eine Fliegerbombe einen Seitenflügel des Hauptgebäudes [7, S. 84]. Die Nachkriegszeit war, wie allerorts, dem Wiederaufbau gewidmet; aufgrund von Materialmangel wurde zwischen den beiden Klinikgebäuden eine Militärbaracke errichtet, um die Platznot zu kompensieren. Priesel starb am 18. November 1955 plötzlich in der Verwaltungsdirektion des Landeskrankenhauses [6, S. 446f.].

\footnotetext{
9 UAl, Personalakt Richard Priesel, Dekan der medizinischen Fakultät an das Ministerium für innere und kulturelle Angelegenheiten (Abt. IV, Erziehung, Kultus und Volksbildung) vom 03.10.1939.
}

\section{Berufung Hans Aspergers und die Innsbrucker Jahre}

Wenige Monate später legte die für Priesels Nachfolge verantwortliche Berufungskommission dem Professorenkollegium einen Dreier- bzw. eigentlich einen Vierervorschlag vor. Dabei erwähnten sie einleitend, dass an den „zukünftigen Kinderkliniker [!] sehr hohe Anforderungen zu stellen" seien, immerhin besaß die „österreichische Pädiatrie, ... einst Weltruf “ ${ }^{10}$

Die Kommission bestand aus dem damaligen Dekan und Gynäkologen Siegfried Tapfer (1900-1981), welcher in der Zeit des Nationalsozialismus als Gynäkologe für die Zwangssterilisierungen an der Universitäts-Frauenklinik „ermächtigt" war, dem HNO-Arzt Ludwig Hörbst, dem Hygieniker Alfred Schinzel, dem Internisten Anton Hittmair (1892-1986), dem Vorstand der Hautklinik Josef Konrad (1900-1979) und dem bereits erwähnten Lang. Letzterer wurde aufgrund seiner Nähe zum NS-Regime zwar 1946 vorübergehend seines Amtes enthoben, jedoch bereits 1948 wieder definitiv in seine Professur eingesetzt [4, S. 145].

In ihrer Bewertung zu Asperger gaben sie zu bedenken, dass dieser ,in seiner fachlichen Entwicklung einseitiger", dafür aber „auf seinem Sondergebiet, der Heilpädagogik, einen über die Ländergrenzen weit hinausreichenden Ruf“ besitze. Die supplierende Leitung

10 Am ehesten bezog sich die Berufungskommission hier auf Theodor Escherich (1857-1911) und Clemens von Pirquet (1874-1929). 
der Wiener Kinderklinik in den ersten drei Nachkriegsjahren wurde ebenfalls erwähnt. Dennoch wurde, die Notwendigkeit eines erfahrenen Klinikers berücksichtigend, Asperger nur an letzter Stelle („tertio loco“) genannt, nach Josef Siegl („primo loco“) bzw. Horst-Günther Krainick (1908-1968) und Adolf Windorfer (1909-1996) („secundo et aequo loco"). Siegl war zu diesem Zeitpunkt „leitender Primararzt" des Mautner Markhof'schen Kinderspitals in Wien, Krainick „Oberarzt der Universitätskinderklinik in Freiburg" und Windorfer der „ärztliche Direktor der Kinderkrankenhäuser in Stuttgart“. Ein weiterer Grund für die Letztreihung Aspergers bestand in der geringen Zahl der PatientInnenbetten, laut dem Vorschlag 70, welche Asperger bzw. der von ihm ausgeübten Heilpädagogik ,auf absehbare Zeit keine Entfaltungsmöglichkeiten" erlauben würde. ${ }^{11}$

Im Kommissionsbericht über Asperger wurde für die Zeit des Zweiten Weltkrieges dessen Habilitation am 27. Juli 1943 und ein Teil seiner Tätigkeiten erwähnt: „Von 1935 bis 1938 in der Mutterberatung [!] in Wien, seit 1938 als Facharzt in Wiener Sonderschulen, ausserdem [!] als Lehrer an Kinderschwesternund Fürsorgerinnenschulen, ausserdem als Gerichtssachverständiger in Wien und Niederösterreich." Asperger hatte zu diesem Zeitpunkt 59 Publikationen vorzuweisen, von denen sich allerdings nur zwölf nicht mit heilpädagogischen Fragen beschäftigten. Interessanterweise geht der Bericht auch auf Aspergers Bewertung von „Vererbungsfragen“ ein, und dass dieser gegenüber den „Möglichkeiten eines Ausgleichs durch die Maßnahmen der Erziehung wie durch ärztliche Maßnahmen“ aufgeschlossen sei. Obwohl Asperger sich einem „Sonderzweig der Kinderheilkunde“ "zugewandt habe, erachtete die Kommission dies als zukunftsträchtige Teildisziplin, denn: „Die Begründung einer Heilpädagogik im Kindesalter entspricht der einzigartigen Gelegenheit, dort vermit-

\footnotetext{
11 UAI, Lehrkanzel für Kinderheilkunde nach Prof. Priesel, Berufungskommission an das Professorenkollegium der medizinischen Fakultät derUniversität Innsbruck vom 09.06.1956.
}

Monatsschr Kinderheilkd 2020 168 (Suppl 3):S197-S203

https://doi.org/10.1007/s00112-020-01014-7

(c) Der/die Autor(en) 2020

\section{Lechner}

\section{Hans Asperger und die Kinderklinik Innsbruck}

\section{Zusammenfassung}

Die Innsbrucker Kinderklinik zog erst 1901 unter ihrem ersten Vorstand Johann Loos in eigenständige Räumlichkeiten; entsprechend prägte Aufbauarbeit die folgenden Jahrzehnte. Auch Loos' Nachfolger Richard Priesel, vormals Schüler zunächst Pirquets und später Hamburgers an der Wiener Kinderklinik, leitete die junge Kinderklinik ab 1935 unter großen organisatorischen und finanziellen Mühen. Der Zweite Weltkrieg verbesserte diese Situation sicherlich nicht. Inwieweit die gemeinsamen Wurzeln an der Wiener Kinderklinik Hans Asperger bei der Berufung als Priesels Nachfolger unterstützten, bleibt unklar. Jedenfalls trat Asperger 1957 als Letztgereihter im Berufungsvorschlag die Nachfolge an. Die Quellenlage für Aspergers fünf Innsbrucker Jahre ist bis auf wenige Unterlagen knapp. Eine systematische Betrachtung steht noch aus, wofür dieser Beitrag hoffentlich einen Anstoß geben mag.

Schlüsselwörter

Richard Priesel · Kinderklinik Innsbruck . Zweiter Weltkrieg · Hans Asperger .

ZeitzeugInnenberichte

\section{Hans Asperger and the Children's Hospital Innsbruck}

\section{Abstract}

The Children's Hospital Innsbruck first moved into its own premises 1911 under its first director Johann Loos and appropriately, setup work characterized the following decades. Loos' successor Richard Priesel, formerly a disciple of initially Pirquet and later Hamburger at the Children's Hospital Vienna, led the young Children's Hospital from 1935 still enduring organizational and financial troubles. World War II did not improve this situation. If the common roots at the Children's Hospital Vienna supported Hans Asperger with his appointment as
Priesel's successor, remains unclear. However, he succeeded Priesel in 1957 despite being the last in the appointment committee's proposal list. The body of source material for Asperger's five years in Innsbruck is, except for a few documents, scarce. A systematic study is pending, which hopefully this article might prompt.

\section{Keywords}

Richard Priesel · Children's Hospital Innsbruck . World War II · Hans Asperger · Contemporary witness reports telnd einzugreifen ..., wo ein Erfolg noch erwartet werden kann. Es sind die Fälle, wo einmal eine hereditäre Anlage Beschränkungen in der Persönlichkeitsentfaltung und Entwicklung begründet, wo zum anderen das Elternhaus entweder nicht zur Verfügung steht oder versagt, wo ferner die Schule nur allzu oft den erziehlichen Notwendigkeiten nicht gerecht wird ...." Die Berufungskommission sprach Asperger abschließend „die Hingabe an das Werk und die grosse Liebe zu den oft anfänglich gar nicht sehr liebenswerten Kindern" ${ }^{\text {zu. }}{ }^{12}$

Die Beweggründe des Ministeriums, schließlich den letztgereihten Asperger

12 UAl, Lehrkanzel für Kinderheilkunde nach Prof. Priesel, Kommissionsbericht zu Hans Asperger.
(• Abb. 3) zum Ordinarius zu ernennen, lassen sich weder aus den Berufungsakten noch dem (sehr dünnen) Personalakt Aspergers eruieren. Die Herkunft aus der Wiener Schule und die damit verbundenen Netzwerke könnten eine gewisse Rolle gespielt haben. Jedenfalls erhielt Asperger mit 23. Februar 1957 den entsprechenden Bescheid. Hittmair, vorheriges Mitglied der Berufungskommission, war zu diesem Zeitpunkt gerade Rektor der Universität Innsbruck und korrespondierte mit Asperger über die Wohnungsfrage. Hieraus wird klar, dass Asperger seine „Familie teils überhaupt nicht ..., teils erst viel später nach Innsbruck transferieren kann“" (s. auch unten). Am 31. März 1957 trat Asperger seinen Dienst an; am 7. Mai hielt er seinen Eröffnungsvortrag zum Thema „Proble- 


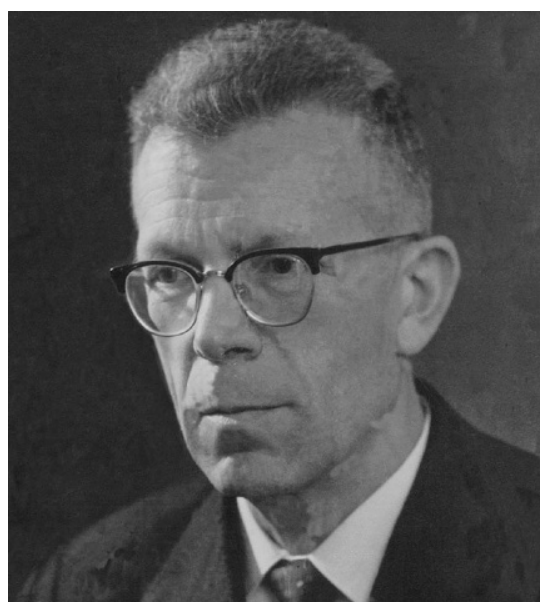

Abb. $3 \Delta$ Porträtfoto von Hans Asperger. (@ Archiv Kinderklinik Innsbruck)

me der modernen Pädiatrie“ [1, S. 109] (• Abb. 4). Bereits wenige Jahre später jedoch, konkret mit 26. Juni 1962, wurde er an die Wiener Kinderklinik berufen, welcher er bis 1977 vorstand. ${ }^{13}$

\section{》) Die Beweggründe des Ministeriums für die Ernennung Aspergers zum Ordinarius sind nicht zu eruieren}

Die klinisch-organisatorischen und wissenschaftlichen Tätigkeiten während der wenigen Innsbrucker Jahre sind aufgrund unzureichender Quellenlage nur bedingt nachvollziehbar. Asperger dürfte die Bemühungen seines Vorgängers um eine Gebäudeerweiterung der Kinderklinik jedenfalls fortgeführt haben, immerhin waren fast 15 Jahre nach Kriegsende immer noch die oben erwähnten Baracken in Verwendung. Ein Erweiterungsbau wurde zwar 1959 begonnen, konnte jedoch erst einige Jahre nach Aspergers Weggang im Jahr 1967 eröffnet werden (• Abb.5).

Sicherlich hat Asperger auch an seinem Lehrbuch Heilpädagogik [2] gearbeitet, denn 1961 wurde schließlich die dritte, überarbeitete und erweiterte Auf-

13 UAl, Personalakt Hans Asperger, Bundesminister für Erziehung, Drimmel an das Dekanat der medizinischen Fakultät der Universität Innsbruck sowie abschriftlich an Hans Asperger vom 28.06.1962.

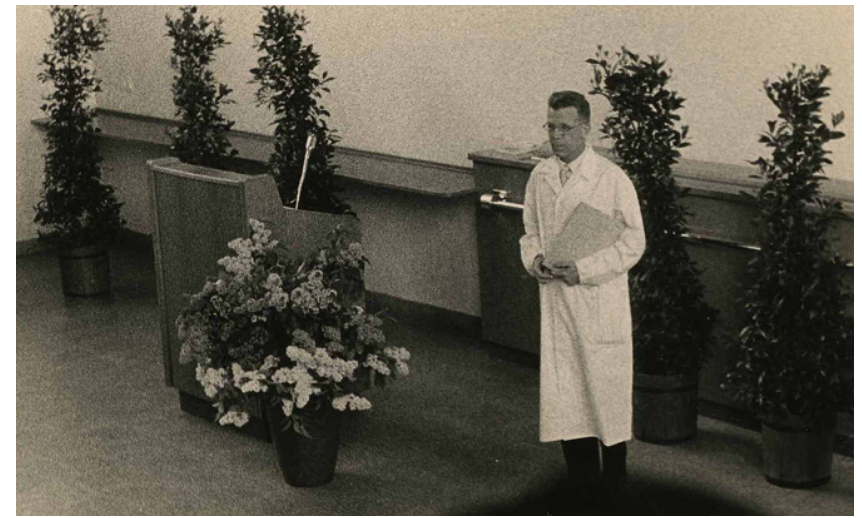

Abb. $4 \varangle$ Hans Asperger beim Eröffnungsvortrag am 07.05.1957. (@ Archiv Maria Asperger Felder)

lage herausgegeben. In der studentischen Lehre hat Asperger neben der üblichen Hauptvorlesung der Pädiatrie auch jedes Semester über „Heilpädagogik. Wege zur Menschenkenntnis" gelesen. ${ }^{14}$ Für die postpromotionelle Weiterbildung wurden Fortbildungen, „pädiatrische klinische Wochenenden", organisiert. Diesbezüglich bestand ein wichtiger Schritt auch in der 1962 erfolgten Gründung der Österreichischen Gesellschaft für Kinderheilkunde, welche zwar von Walter Swoboda (1915-2008) initiiert, aber von Anfang an durch Asperger sehr unterstützt wurde [9, S. 43].

Zum in Erinnerung gebliebenen Auftreten an der Kinderklinik selbst stehen unten stehende Berichte von ZeitzeugInnen zur Verfügung. Diese können ergänzt werden durch die Einschätzung des Innsbrucker Historikers Franz Huter (1899-1997), dass „Asperger sich ... rasch einen Kreis von Freunden und Fachkollegen schaffen"konnte [6, S. 447].

\section{Erzählungen zu Hans Aspergers Zeit in Innsbruck}

Dieser Beitrag möchte neben der Wiedergabe von im Universitätsarchiv bzw. der angegebenen Literatur gefundenen Informationen die Innsbrucker Jahre Aspergers auch aus der Sicht von ZeitzeugInnen abbilden, erhebt hierbei aber natürlich keinen Anspruch auf Vollständigkeit. Wie auch im Beitrag „Individuelle Erfahrungsberichte von SchülerInnen und KollegInnen Hans Aspergers" in diesem Sonderheft sowie

14 UAI, Vorlesungsverzeichnisse WS 1957/1958 bis SS 1962. beim Online-Zusatzmaterial zu berücksichtigen, sind persönliche Berichte von ZeitzeugInnen immer kritisch zu reflektieren. Zum einen ist der zeitliche Abstand zu den geschilderten Geschehnissen bereits ein sehr großer, zum anderen mögen persönliche Interessen bei der Nacherzählung von Geschichten eine gewisse Rolle spielen. Die für diesen Abschnitt kontaktierten Personen sind sich auf Hinweis des Autors dieser Einschränkungen bewusst. ${ }^{15}$

Als Hans Asperger 1957 nach Innsbruck berufen wurde, wollte seine Ehefrau Hanna (geb. Kalmon, 1909-1998) lieber in Wien bleiben, immerhin war sie dort bereits seit vielen Jahren heimisch. Die beiden hatten sich letztlich durch einen Zufall kennengelernt: Die im westfälischen Hiddingsel als 12. Kind ihrer Eltern aufgewachsene Hanna Kalmon wollte eigentlich nur für ein Semester ihres in Münster begonnenen Sportstudiums nach Wien kommen, um besonders ihre Wintersportfähigkeiten zu verbessern. Ihr bekannte Theologen (oder Theologiestudenten), jedenfalls Mitglieder beim Bund Neuland, wollten sie bei der Ankunft in Wien abholen, waren aber verhindert und baten ihren Neuländer Kollegen Hans Asperger, ihre Bekannte Hanna am Bahnhof abzuholen.

\footnotetext{
15 Herzlich gedankt sei an dieser Stelle Dr. Gertrud Schaafhausen, geb. Asperger (*1936), Dr. Maria Asperger Felder (*1946), Dr. Reingard Ciresa $\left({ }^{*} 1938\right)$, ao. Univ.-Prof. Dr. Hubert Haberfellner $\left({ }^{*} 1941\right)$, Dr. Erna Jaschke $\left({ }^{*} 1942\right)$, ao. Univ.-Prof. Dr. Edwin Knapp (*1940), Agnes Leitgeb (geb. Oberdorfer, * 1939), Maria Mayr

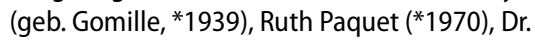
Christine Scheminzky $\left.{ }^{*} 1937\right)$ und Waltraud Unterasinger (geb. Oberdorfer, ${ }^{*} 1941$ ).
} 


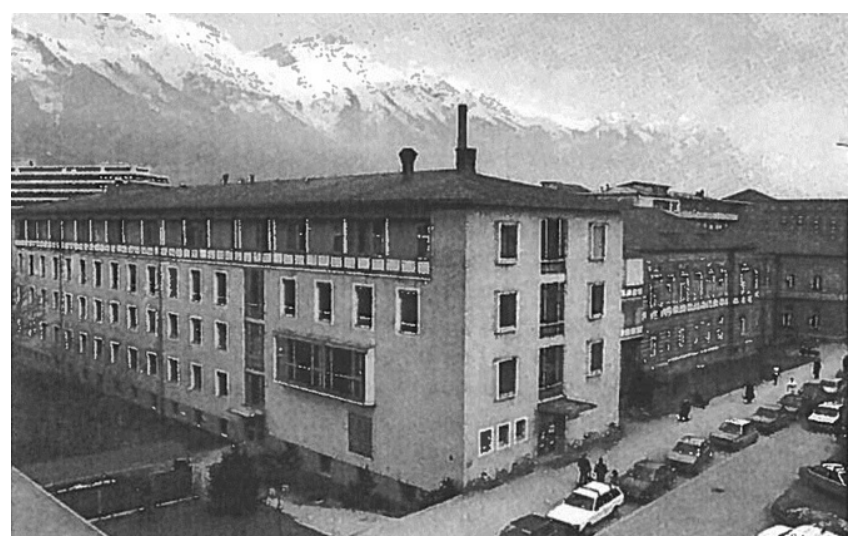

Abb. 5 A Ansicht des Erweiterungsbaus von einem Haus in der Innerkoflerstraße im Südwesten, um 1970. (@ Archiv Kinderklinik Innsbruck)

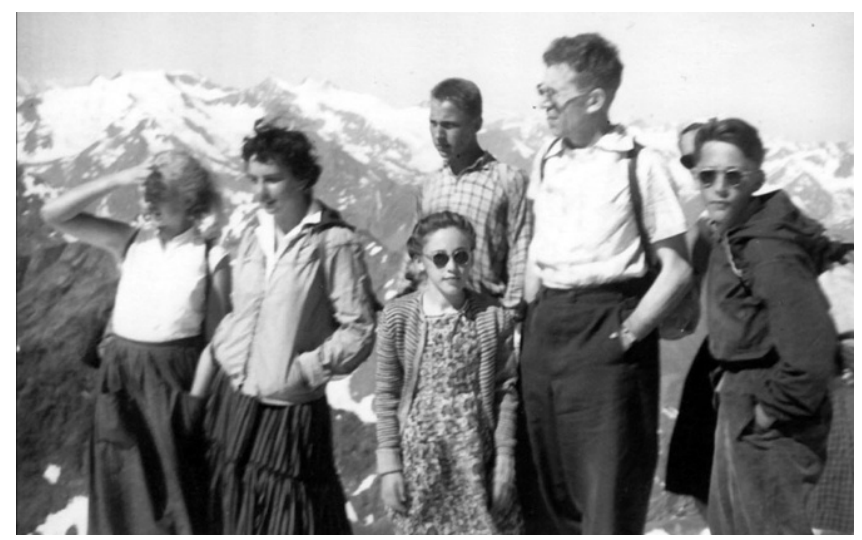

Abb. $6 \Delta$ Das Foto zeigt Asperger 1957 am Habicht in den Stubaier Alpen. (๑) Maria Leitgeb, selbst ganz links im Bild, die das Foto freundlicherweise zur Verfügung gestellt hat)
Die beiden wurden in der Folge ein Paar und heirateten 1935. Asperger selbst erzählte hierüber in einem Radiointerview: „Beim Bergsteigen habe ich auch meine Frau gefunden, kennengelernt, wirklich. Sie ist die Frau meines Lebens gewesen und geblieben“([3], min. 35-35). Aus der Ehe gingen fünf Kinder hervor: Gertrud $\left({ }^{\star} 1936\right)$, Hans $\left({ }^{\star} 1938\right)$, Hedwig $\left({ }^{\star} 1940\right)$, Maria $\left({ }^{\star} 1946\right)$ und Brigitte $\left({ }^{\star} 1948\right)$ [1, S. 115].

Auch die Kinder Aspergers, entweder in der Schule oder an der Universität in Ausbildung, verblieben zunächst in Wien. Die älteste Tochter Gertrud folgte dem Vater aber bereits wenig später nach Innsbruck und setzte dort ihr Medizinstudium im 7. Semester fort. Zunächst wohnten Vater und Tochter getrennt voneinander, der Vater schlief wohl in seinem Vorstandszimmer in der Kinderklinik, die Tochter war anderweitig untergebracht. Nach wenigen Monaten ergab sich allerdings die Gelegenheit zum Zusammenziehen in einer Parterrewohnung. Tochter Gertrud kümmerte sich neben ihrem Studium auch um den Haushalt. Die Jahre in dieser Wohnung und überhaupt in Innsbruck selbst hat Gertrud Asperger als „sehr schön“ in Erinnerung. ${ }^{16}$ Die Wohnung lag in der Innsbrucker Kärntnerstraße und die Vermietung (oder zumindest Vermittlung) geschah möglicherweise über die Fakultät bzw. Universität, denn bereits

16 Telefonische Mitteilung von Gertrud Schaafhausen vom 24.04.2020. vor ihnen hatte ein "Professor" dort gelebt. Im Sommer wurden die Fahrräder genutzt, um zur Klinik bzw. zur Uni zu gelangen; ein begeisterter Radfahrer war Hans Asperger jedoch nie. Im Winter nahmen Vater und Tochter öfter das Auto, einen Mercedes, um durch die Stadt zu fahren. Im Jahr 1961 promovierte Gertrud Asperger in Innsbruck; das letzte Mal vor Ort war sie 2011 für das Goldene (also 50-jährige) Doktorjubiläum.

Alle Geschwister schlossen ein Hochschulstudium ab: Dr. Brigitte Jedelsky (geb. Asperger) ist, nach einem Studium an der Universität für Bodenkultur Wien (BOKU), Landschaftsplanerin geworden und lebt in Wien. Hans jun. absolvierte ebenfalls ein Studium an der BOKU. Hedwig Lütke Entrup (geb. Asperger) studierte auch an der BOKU, war als Landwirtin tätig und bewirtschaftet weiterhin den von mütterlicher Seite erhaltenen Hof im westfälischen Hiddingsel. ${ }^{17}$ Dort hat sie sich auch als Vertreterin des Landfrauenverbandes engagiert. ${ }^{18}$ Maria Asperger Felder trat in die Fußstapfen des Vaters, studierte ebenfalls Medizin und ist als Kinder- und Jugendpsychia-

\footnotetext{
17 Mitteilung von Ruth Paquet vom 10.08.2020.

18 Protokoll der Dorfversammlung vom 14.04.2011; https://bit.ly/3fFD6Zx, zugegriffen am 08.08.2020.
}

terin mit dem Schwerpunkt Autismus in Zürich tätig. ${ }^{19}$

Während seiner Innsbrucker Jahre verbrachte Hans Asperger zumindest jedes zweite Wochenende bei der Familie in Wien und hielt dort dann wohl auch Ordination. Auch Gertrud Asperger begleitete den Vater oft nach Wien. Die Wochenenden in Innsbruck bzw. Tirol auf der anderen Seite waren geprägt von Bergsteigen, Wandern und Skifahren gemeinsam mit anderen Neuländern, immerhin waren Aspergers Kinder ebenfalls alle Mitglieder.

\section{》) Über die Teilnahme an Aktivitäten des Bund Neuland waren Asperger und seine Tochter sozial gut vernetzt}

Zahlreiche Gipfel im Ziller- und Stubaital sowie im Südtiroler Ortlergebiet wurden während dieser Jahre bestiegen (- Abb. 6). Einer der bergsteigerischen Höhepunkte war die Besteigung des Matterhorns 1961 durch Vater und Tochter Asperger gemeinsam mit Adolf Unterasinger $\left({ }^{\star} 1931\right)$ und Bernhard Haffner (1939-2002). Letzterer war ebenfalls an der Kinderklinik tätig und leitete dort später die von ihm begründete EEG-Ambulanz.

\footnotetext{
19 "Es ist keine Krankheit, sondern eher eine Besonderheit", 25.05.2019; https://bit.ly/ 2PwFw2a, zugegriffen am 08.08.2020.
} 
Über diese rege Teilnahme an den Aktivitäten des Bund Neuland waren Vater und Tochter auch sozial gut vernetzt und lernten hierbei u.a. die spätere Dermatologin Erna Jaschke $\left({ }^{\star} 1942\right)$, Waltraud Unterasinger $\left({ }^{*} 1941\right)$ und Werner Maleczek $\left({ }^{\star} 1944\right)$ kennen. Gertrud Asperger hat mit den jüngeren Neuländern auch regelmäßig ohne den Vater Ausflüge unternommen und, obwohl größtenteils Bus und Bahn zur Fortbewegung genutzt wurden, hin und wieder auch das Auto des Vaters verwenden dürfen. Viele Neuländer waren in diesen Jahren zeitgleich auch Mitglieder bei der Jugendgruppe der Sektion Innsbruck des Österreichischen Alpenvereins. ${ }^{20}$ Die Verbundenheit der Tiroler Neuländer mit Hans Asperger zeigte sich auch darin, dass Waltraud Unterasinger und Bernhard Haffner gewissermaßen als offizielle Abordnung an Aspergers Begräbnis 1980 in Wien teilnahmen.

\section{》) Aspergers zurückhaltender \\ Führungsstil hat „sehr viel Freiheit" gelassen}

An der Kinderklinik selbst bzw. der Fakultät sei Asperger recht beliebt gewesen, offenbar sei nämlich in den Jahren des "Interregnums" bzw. auch während der letzten Jahre unter der Ägide Priesels die Atmosphäre an der Klinik keine sehr gute gewesen. Asperger habe ein „lockeres und verspieltes" Klima etablieren können und von Anfang an die Meinungen aller GesprächspartnerInnen geschätzt, unabhängig davon, ob diese ebenfalls ProfessorInnen oder Krankenschwesternschülerinnen waren..$^{21}$ Besonders in Erinnerung blieb offenbar auch, dass Asperger gute Arbeit auffallend oft auch aktiv gelobt habe. Sein zurückhaltender Führungsstil "lässt sehr viel Freiheit" und wurde deswegen teils auch kritisiert, „weil man meint, er dirigiere zu wenig“. Asperger selbst glaubte dadurch eine „Atmosphäre der Arbeit und Zusammenarbeit" $\mathrm{zu}$ schaffen und freute sich „über jede

\footnotetext{
20 Mitteilung von Erna Jaschke vom 09.04.2020.

21 Telefonische Mitteilungen von Agnes Leitgeb
} vom 24.04.2020.
Selbstständigkeit, jede wissenschaftliche“" ([3], min. 45-47).22

Auch mit den Kindern und Jugendlichen habe sich Asperger auf eine Art und Weise unterhalten, wie sie vielen an der Kinderklinik eine neue gewesen sein soll. Er habe diese als in Entstehung befindliche Persönlichkeiten erachtet und wertgeschätzt und ihnen immer mit Ernsthaftigkeit zugehört. An der Innsbrucker Kinderklinik seien die AutistInnen Aspergers „Lieblingspatienten“ gewesen. Regelmäßig sei Asperger zudem am Sonntagabend nach dem Wandern oder Bergsteigen an der Klinik vorbeigekommen, nicht unbedingt, um nach dem Rechten zu sehen, sondern um sich mit den PatientInnen auszutauschen und mit ihnen zu spielen. Asperger habe, wenn sich die PatientInnen im Gespräch mit ihm als besonders erwiesen haben, diese auch etwa im Sinne von „du bist ein Gescheiter“ väterlich gelobt. ${ }^{23}$

Auch aus einem früheren Patientenbericht geht hervor, dass Asperger seine ihm gegenübersitzenden PatientInnen ernst genommen und ihnen wirklich zugehört habe. Wenn er nach solchen teils „vergnüglichen Unterredungen“ keinen Grund zu weiterer Diagnostik und Therapie sah, habe er dennoch gern weitere Gespräche angeboten, „wann es nur möglich sei“ 24

\section{\) Aspergers Wertschätzung ihrer Arbeit wurde von den Krankenschwestern als etwas Neues empfunden}

Im Unterricht habe Asperger, wie damals noch üblicher als heute, den Studierenden gern PatientInnen vorgestellt. Dies geschah aus Sicht der Studierenden auf eine „ruhige, geduldige, rück-

${ }^{22}$ Vgl. Zeitzeugenbericht von J. Deutsch im E-Supplement.

${ }^{23}$ Telefonische Mitteilungen von Agnes Leitgeb und Maria Mayr-Gomille vom 24.04.2020. Die beiden langjährigen Kinderkrankenschwestern hatten zwischen 1956 und 1959 ihr Diplom gemacht und dieses entsprechend unter Asperger abgeschlossen.

${ }^{24}$ Mitteilung von Hubert Haberfellner vom 25.04.2020. sichtsvolle und vor allem empathische Weise“. ${ }^{25}$ Aspergers katholische Einstellung sei ebenfalls sehr spürbar gewesen, nach der Vorstellung von schwer kranken Kindern habe Asperger deren Schicksal mitfühlend, nicht abwertend kommentiert, mit „das habe der liebe Herrgott aber nicht gut gemacht. “26 Die Vorlesungen selbst seien wohl hinsichtlich ihrer pädiatrisch-inhaltlichen Qualität unter jenen des nach Asperger die Lehre übernehmenden Hans Rösslers gewesen. Den Studierenden sei von Asperger eine Wertschätzung der PatientInnen und die Aneignung einer humanitären Weltanschauung vermittelt worden. Das pädiatrische Wissen wurde eher durch Studium der empfohlenen Literatur, wie etwa des vom bekannten österreichischen Pädiater Meinhard von Pfaundler (1872-1947) etablierten Lehrbuchs [5], erworben. ${ }^{27}$

Auch den Unterricht für die Kinderkrankenschwesternschülerinnen habe Asperger wohl sehr gern gemacht, zumindest empfanden das damals in Ausbildung befindliche Krankenschwestern so. Vor allem die Wertschätzung ihrer wichtigen Arbeit bei der Therapie der kleinen PatientInnen sei für viele damals etwas Neues gewesen, was die Stimmung auf den Diplomfeiern entsprechend positiv beeinflusst habe (• Abb. 7). ${ }^{28}$

\section{Schlussbemerkungen}

Die Geschichte der Innsbrucker Kinderklinik vor Asperger sowie dessen Berufungsverfahren zu betrachten, ermöglicht einen gewissen Blick auf die Voraussetzungen, auf welche Asperger 1957 in Innsbruck traf. Mit Priesel war dort nicht nur ein ehemaliger Kollege, sondern ebenfalls ein Hamburger-Schüler über zwanzig Jahre Ordinarius. Nach-

\footnotetext{
${ }^{25}$ Vgl. Zeitzeugenbericht von I. Hammerer im E-Supplement.

${ }^{26}$ Mitteilung von Edwin Knapp vom 30.03.2020. $\mathrm{Vgl}$. auch Zeitzeugenbericht von 0 . Jürgenssen in diesem Heft.

27 Telefonische Mitteilungen von Reingard Ciresa und Edwin Knapp vom 30.03.2020.

28 Telefonische Mitteilungen von Agnes Leitgeb und Maria Mayr-Gomille vom 24.04.2020.
} 


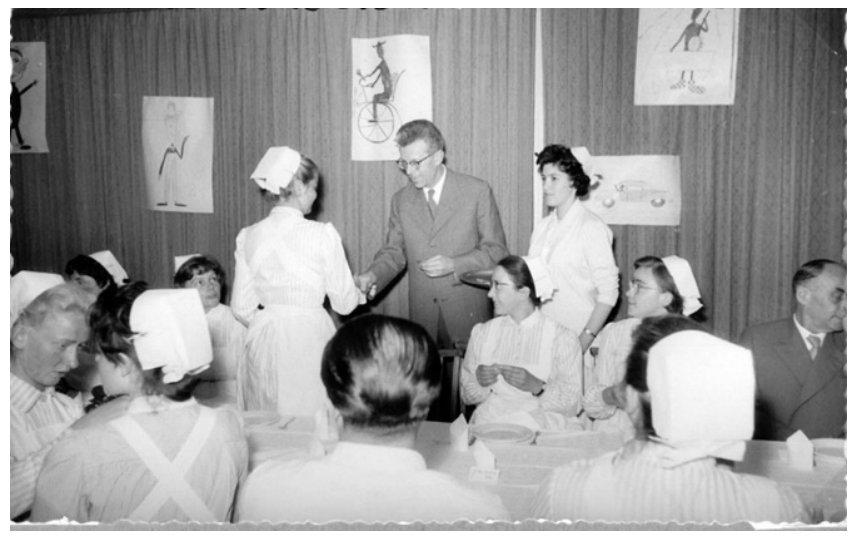

Abb. $7 \triangleleft$ Asperger bei der Diplomverleihung 1959 (৫) Maria Leitgeb, die das Foto freundlicherweise zur Verfügung gestellt hat)

dem die Kinderklinik aber erst 1901 eröffnet wurde, hatte Priesel vor und nach dem Zweiten Weltkrieg noch einiges an Aufbauarbeit zu leisten.

Asperger konnte diese fortsetzen und u. a. eine Gebäudeerweiterung initiieren, deren Abschluss jedoch erst nach seiner Zeit erfolgte. Inwieweit sich Asperger, trotz seiner Begeisterung für das Wandern und Bergsteigen, langfristig in Innsbruck sah, bleibt spekulativ; bis auf seine älteste Tochter hat er seine Familie jedenfalls in Wien belassen. Eventuelle kinderklinikinterne, zukunftsträchtige Innovationen sind aus den eingesehenen Quellen nicht ableitbar. Eine systematische Betrachtung dieser Jahre steht allerdings noch aus, wofür dieser kurze Beitrag einen Anstoß geben mag.

\section{Korrespondenzadresse}

\section{Mag. Dr. C. Lechner}

Department für Kinder- und Jugendheilkunde, Pädiatrie I, Medizinische Universität Innsbruck Anichstr. 35, 6020 Innsbruck, Österreich ch.lechner@i-med.ac.at

Mag. Dr. C. Lechner, geb. 1987. Studium der Humanmedizin in Innsbruck und der Geschichtswissenschaften in Innsbruck, New Orleans und Heidelberg mit Schwerpunkt auf Medizin- und Wissenschaftsgeschichte. Assistenzarzt für Kinder- und Jugendheilkunde am Department für Kinder- und Jugendheilkunde Innsbruck seit Mai 2015. Wissenschaftliche Tätigkeiten auf dem Gebiet der Neuropädiatrie und Neuroimmunologie sowie der Geschichte von Pädiatrie und Anatomie.

Funding. Open access funding provided by University of Innsbruck and Medical University of Innsbruck.

\section{Einhaltung ethischer Richtlinien}

Interessenkonflikt. C. Lechner gibt an, dass kein Interessenkonflikt besteht.

Für diesen Beitrag wurden vom Autor keine Studien an Menschen oder Tieren durchgeführt. Für die aufgeführten Studien gelten die jeweils dort angegebenen ethischen Richtlinien.

Open Access Dieser Artikel wird unter der Creative Commons Namensnennung 4.0 International Lizenz veröffentlicht, welche die Nutzung, Vervielfältigung, Bearbeitung, Verbreitung und Wiedergabe in jeglichem Medium und Format erlaubt, sofern Sie den/die ursprünglichen Autor(en) und die Quelle ordnungsgemäßnennen, einen Link zur Creative Commons Lizenz beifügen und angeben, ob Änderungen vorgenommen wurden.

Die in diesem Artikel enthaltenen Bilder und sonstiges Drittmaterial unterliegen ebenfalls der genannten Creative Commons Lizenz, sofern sich aus der Abbildungslegende nichts anderes ergibt. Sofern das betreffende Material nicht unter der genannten Creative Commons Lizenz steht und die betreffende Handlung nicht nach gesetzlichen Vorschriften erlaubt ist, ist für die oben aufgeführten Weiterverwendungen des Materials die Einwilligung des jeweiligen Rechteinhabers einzuholen.

Weitere Details zur Lizenz entnehmen Sie bitte der Lizenzinformation auf http://creativecommons.org/ licenses/by/4.0/deed.de.

\section{Literatur}

1. Asperger Felder M (2008) „Zum Sehen geboren, zum Schauen bestellt“ Hans Asperger (1906-1980: Leben und Werk). In: Castell R (Hrsg) Hundert Jahre Kinder- und Jugendpsychiatrie. Biografien und Autobiografien. V\&R unipress, Göttingen, $S$ 99-117

2. Asperger H (1961) Heilpädagogik. Einführung in die Psychopathologie des Kindes für Ärzte, Lehrer, Psychologen, Richter und Fürsorgerinnen. Springer, Wien

3. Asperger H (1978) Geschichten und Geschichte - Autobiographische Aussagen von Hans Asperger. Radiointerview mit Hans Asperger vom 24.12.1974. https://www.mediathek.at/atom/ 01782B10-0D9-00CD5-00000BEC-01772EE2.Zugegriffen:24. März 2020
4. Goller P, Tidl G (2012) „Jubel ohne Ende...!" Die Universität Innsbruck im März 1938. Zur Nazifizierung der Tiroler Landesuniversität. Erhard Löcker, Wien

5. Husler J (1955) Lust-Pfaundler-Husler Krankheiten des Kindesalters. Ihre Erkennung und Behandlung in der Praxis. Urban \& Schwarzenberg, München, Berlin

6. HuterF (1969) Hundert Jahre Medizinische Fakultät Innsbruck 1869 bis 1969. 2. Teil: Geschichte der Lehrkanzeln, Institute und Kliniken (Universität Innsbruck)

7. Pfannerstill A (2001) Die Baugeschichte der Klinik Innsbruck. Vom Neuen Stadtspital zum a.ö. Landeskrankenhaus 1885-1948 (Diplomarbeit). Innsbruck

8. Seidler E (2007) Jüdische Kinderärzte 1933-1945. Entrechtet/Geflohen/Ermordet. Karger, Basel, Freiburg

9. Sperl W, Kerbl R (Hrsg) (2012) 50 Jahre ÖGKJ. Festschrift. Holzhausen, Wien

10. Wagner R (1925) Die Behandlung der kindlichen Zuckerharnruhr. Springer, Berlin, Heidelberg, New York 